\title{
On the Edge Metric Dimension of Different Families of Möbius Networks
}

\author{
Bo Deng, ${ }^{1,2}$ Muhammad Faisal Nadeem $\mathbb{D}^{3},{ }^{3}$ and Muhammad Azeem $\mathbb{D}^{3,4}$ \\ ${ }^{1}$ School of Mathematics and Statistics, Qinghai Normal University, Xining, China \\ ${ }^{2}$ Academy of Plateau Science and Sustainability, Xining, Qinghai 810008, China \\ ${ }^{3}$ Department of Mathematics, COMSATS, University Islamabad Lahore Campus, Lahore, Pakistan \\ ${ }^{4}$ Department of Aerospace Engineering, Faculty of Engineering, Universiti Putra Malaysia, Seri Kembangan, Malaysia \\ Correspondence should be addressed to Muhammad Faisal Nadeem; mfaisalnadeem@ymail.com
}

Received 20 December 2020; Revised 27 February 2021; Accepted 1 March 2021; Published 25 March 2021

Academic Editor: Ali Ahmad

Copyright $\odot 2021$ Bo Deng et al. This is an open access article distributed under the Creative Commons Attribution License, which permits unrestricted use, distribution, and reproduction in any medium, provided the original work is properly cited.

\begin{abstract}
For an ordered subset $Q_{e}$ of vertices in a simple connected graph $G$, a vertex $x \in V$ distinguishes two edges $e_{1}, e_{2} \in E$, if $d\left(x, e_{1}\right) \neq d\left(x, e_{2}\right)$. A subset $Q_{e}$ having minimum vertices is called an edge metric generator for $G$, if every two distinct edges of $G$ are distinguished by some vertex of $Q_{e}$. The minimum cardinality of an edge metric generator for $G$ is called the edge metric dimension, and it is denoted by $\operatorname{dim}_{e}(G)$. In this paper, we study the edge resolvability parameter for different families of Möbius ladder networks and we find the exact edge metric dimension of triangular, square, and hexagonal Möbius ladder networks.
\end{abstract}

\section{Introduction}

A simple connected graph $G=(V, E)$ with vertex set and edge set $V$ and $E$, respectively. For two vertices $a_{1}, a_{2} \in V$, the distance $d\left(a_{1}, a_{2}\right)$ between vertices $a_{1}$ and $a_{2}$ is the count of edges between them. A vertex $v \in V$ is said to distinguish two vertices $a_{1}$ and $a_{2}$, if $d\left(v, a_{1}\right) \neq d\left(v, a_{2}\right)$. A set $Q \subset V$ is called a resolving set of $G$, if any pair of distinct vertices of $G$ is distinguished by some element of $Q$. A resolving set of minimum cardinality is named as metric basis, and its cardinality is the metric dimension of $G$, denoted by $\operatorname{dim}(G)$. A vertex $v \in V$ and an edge $e=a_{1} a_{2} \in E$, and the distance between $v$ and $e$ is defined as $d(e, v)=\min \left\{d\left(a_{1}, v\right), d\left(a_{2}, v\right)\right\}$. A vertex $x \in V$ distinguishes two edges $e_{1}, e_{2} \in E$, if $d\left(x, e_{1}\right) \neq d\left(x, e_{2}\right)$. A subset $Q_{e}$ having minimum vertices from a connected graph $G$ is an edge resolving set for $G$, if every two distinct edges of $G$ are distinguished by some vertex of $Q_{e}$. The minimum cardinality of an edge resolving set for $G$ is called the edge metric dimension and is denoted by $\operatorname{dim}_{e}(G)$.

In 1975, the idea of metric dimension was delivered by Slater [1], he named the metric generators as locating sets which relates to the problem of uniquely recognizing the position of intruders in networks. On the same idea, in 1976 the concept of metric dimension of a graph was independently introduced by Harary and Melter in [2], and these time metric generators were named as resolving sets. One can think that instead of distinguishing two distinct vertices of graph according to chosen subset of vertices, two edges can be distinct with the same subset of vertices. For this concept, Kelenc et al. [3] introduced a new parameter named as the edge metric dimension. In this, they used graph metric to identify each pair of edges by the distance of graph to a chosen subset of vertices.

As far as the idea of metric dimension is extensively studied and used in different fields of science as applications, Chartrand et al. in [4] relate the metric dimension of graph with the drug discovery and pharmacological activity, Khuller et al. in [5] try to put thinking that a robot can be shifted from Euclidean space to graph structure and left his thought as an application of metric dimension in robot navigation, and the metric dimension of Hamming graphs leads Chvátal to the analysis of mastermind games and opens the doors for researchers to view application of metric dimension in complex digital games [6], and in [7, 8], Erdös and Lindström assume that the metric dimension can be 


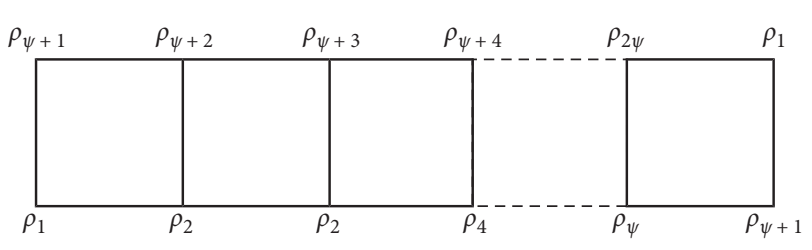

FIgURE 1: Möbius ladder network $\mathrm{ML}_{\Psi}$.

used in various coin-weighing problems. Resolving sets have served as inspiration for many theoretical studies of graphs.

The edge metric dimension is the natural generalization of resolving set, and readers are directed towards the interesting literature containing the metric dimension of different classes of graphs for example, Ali et al. [9, 10] studied the metric dimension of Möbius networks and some other cycle-related graphs, Kuziak et al. [11] discussed the strong metric dimension of graphs, Liu et al. [12, 13] discussed the metric dimension of cocktail party, Toeplitz, and jellyfish graphs, and some cycle-related graphs were studied with the concept of metric dimension by Ahmad et al. [14]. Recently, the edge metric dimension becomes a very common topic in resolvability and a lot of families of graphs are studied. Koam and Ahmad [15] studied edge metric dimension of barycentric subdivision of Cayley graph. The convex polytope graph was discussed by Zhang and Gao in [16] and Ahsan et al. in [17]. Yang et al. discussed some chemical structures related to wheel graphs in [18]. Raza and Bataineh did comparative analysis between metric and edge metric dimension in [19]. Moreover, some interesting study of edge metric dimension can be found in [20, 21], where Okamoto et al. studied the local metric dimension and Yero briefly discussed the definition of metric and its related concepts. Mixed metric dimension is another type of dimension which satisfies the conditions of metric and edge metric dimensions simultaneously. Mixed metric dimension of different families of graphs is studied and gives their exact values, such as Raza et al. studied the mixed metric dimension different rotationally symmetric graphs and gave their exact values [22], Raza and Ji computed the mixed metric dimension of the generalize Petersen graph $P(n, 2)$ [23], and results on mixed metric dimension of some pathrelated graphs are discussed by Raza et al. in [24].

In general, the edge metric dimension of a graph is NPhard [3]. There is no general relation between metric and edge metric dimension of graphs but, in [3], Kelenc et al. inquired about the families of graphs which have $\operatorname{dim}(G)=\operatorname{dim}_{e}(G)$, $\operatorname{dim}(G)<\operatorname{dim}_{e}(G)$, and $\operatorname{dim}(G)>\operatorname{dim}_{e}(G)$. In this paper, we find the edge metric dimension of different families of Möbius ladder networks and give a comparison between the metric and edge metric dimension of these families, in the response of this question.

Definition 1. The minimum number of edges $h$ between two vertices $a_{1}, a_{2}$ of a cycle (sub) graph is called as $h$-size gap between $a_{1}, a_{2}$.

\section{Edge Metric Dimension of Möbius Ladder Network}

Möbius ladder $\mathrm{ML}_{\psi}$ is built by a grid of $\psi \times 1$, and this grid is twisted at $180^{\circ}$; now, paste the extreme most left and right paths of vertices as seen in Figure 1. It contains $\psi$-horizontal cycles of order four. The metric dimension of $\mathrm{ML}_{\psi}$ is three [9], and in our first result, we prove that the edge metric dimension of $\mathrm{ML}_{\psi}$ is four.

Theorem 1. Let $M L_{\psi}$ be a Möbius ladder network with $\psi \geq 3$. Then,

$$
\operatorname{dim}_{e}\left(M L_{\psi}\right)=4
$$

Proof. Consider the edge resolving set $Q_{e}=\left\{\rho_{1}, \rho_{2}\right.$, $\left.\rho_{\lfloor(\psi+3 / 2)\rfloor}, \rho_{\psi+1}\right\}$, to prove that the $\operatorname{dim}_{e}\left(\mathrm{ML}_{\psi}\right)=4$, first of all, we show that $\operatorname{dim}_{e}\left(\mathrm{ML}_{\psi}\right) \leq 4$, and for this claim, following are the distances of all edges with respect to edge resolving set:

$$
\begin{aligned}
d\left(\rho_{\omega} \rho_{\psi+\omega}, \rho_{1}\right)= \begin{cases}\omega-1, & \text { if } \left.\omega=1,2, \ldots, \mid \frac{\psi+2}{2}\right\rfloor, \\
\left\lfloor\frac{\psi+3}{2}\right\rfloor-\omega+\left\lfloor\frac{\psi-1}{2}\right\rfloor, & \text { if } \omega=\left\lfloor\frac{\psi+3}{2}\right\rfloor, \ldots, \psi,\end{cases} \\
d\left(\rho_{\omega} \rho_{\omega+1}, \rho_{1}\right)= \begin{cases}\omega-1, & \text { if } \left.\omega=1,2, \ldots, \mid \frac{\psi+2}{2}\right\rfloor ; \\
\left\lfloor\frac{\psi+4}{2}\right\rfloor-\omega+\left\lfloor\frac{\psi-1}{2}\right\rfloor, & \text { if } \omega=\left\lfloor\frac{\psi+4}{2}\right\rfloor, \ldots, \psi .\end{cases} \\
d\left(\rho_{\psi+\omega} \rho_{\psi+\omega+1}, \rho_{1}\right)= \begin{cases}\omega, & \text { if } \left.\omega=1,2, \ldots, \mid \frac{\psi}{2}\right\rfloor ; \\
\left\lfloor\frac{\psi-1}{2}\right\rfloor-\omega+\left\lfloor\frac{\psi+2}{2}\right\rfloor, & \text { if } \omega=\left\lfloor\frac{\psi+2}{2}\right\rfloor, \ldots, \psi-1 .\end{cases}
\end{aligned}
$$




$$
\begin{aligned}
& d\left(\rho_{2 \psi} \rho_{1}, \rho_{1}\right)=0, \\
& d\left(\rho_{2 \psi} \rho_{1}, \rho_{2}\right)=2, \\
& d\left(\rho_{2 \psi} \rho_{1}, \rho_{\lfloor(\psi+3 / 2)\rfloor}\right)=\left\lfloor\frac{\psi}{2}\right\rfloor, \\
& d\left(\rho_{\omega} \rho_{\psi+\omega}, \rho_{2}\right)= \begin{cases}1, & \text { if } \omega=1 ; \\
\omega-2, & \text { if } \omega=2,3, \ldots,\left\lfloor\frac{\psi+5}{2}\right\rfloor \\
\left\lfloor\frac{\psi}{2}\right\rfloor-\omega+\left\lfloor\frac{\psi+7}{2}\right\rfloor, & \text { if } \omega=\left\lfloor\frac{\psi+7}{2}\right\rfloor \ldots, \psi .\end{cases} \\
& d\left(\rho_{\omega} \rho_{\omega+1}, \rho_{2}\right)= \begin{cases}0, & \text { if } \omega=1 ; \\
\omega-2, & \text { if } \omega=2,3, \ldots,\left\lfloor\frac{\psi+4}{2}\right\rfloor ; \\
\left\lfloor\frac{\psi+6}{2}\right\rfloor-\omega+\left\lfloor\frac{\psi-1}{2}\right\rfloor, & \text { if } \omega=\left\lfloor\frac{\psi+6}{2}\right\rfloor, \ldots, \psi .\end{cases} \\
& d\left(\rho_{\psi+\omega} \rho_{\psi+\omega+1}, \rho_{2}\right)= \begin{cases}1, & \text { if } \omega=1 ; \\
\omega-1, & \text { if } \omega=2,3, \ldots,\left\lfloor\frac{\psi+3}{2}\right\rfloor ; \\
\left\lfloor\frac{\psi+5}{2}\right\rfloor-\omega+\left\lfloor\frac{\psi-1}{2}\right\rfloor, & \text { if } \omega=\left\lfloor\frac{\psi+5}{2}\right\rfloor, \ldots, \psi-1 .\end{cases} \\
& d\left(\rho_{\omega} \rho_{\psi+\omega}, \rho_{\lfloor\lfloor\psi+3 / 2\rfloor}\right)= \begin{cases}\left\lfloor\frac{\psi}{2}\right\rfloor, & \text { if } \omega=1 \\
\left\lfloor\frac{\psi-1}{2}\right\rfloor-\omega+2, & \text { if } \omega=2,3, \ldots,\left\lfloor\frac{\psi+3}{2}\right\rfloor \\
\omega-\left\lfloor\frac{\psi+5}{2}\right\rfloor+1, & \text { if } \omega=\left\lfloor\frac{\psi+5}{2}\right\rfloor, \ldots, \psi\end{cases} \\
& d\left(\rho_{\omega} \rho_{\omega+1}, \rho_{\lfloor\psi+3 / 2\rfloor}\right)= \begin{cases}\left\lfloor\frac{\psi-1}{2}\right\rfloor-\omega+1, & \text { if } \omega=1,2, \ldots,\left\lfloor\frac{\psi+1}{2}\right\rfloor \\
\omega-\left\lfloor\frac{\psi+3}{2}\right\rfloor, & \text { if } \omega=\left\lfloor\frac{\psi+3}{2}\right\rfloor, \ldots, \psi,\end{cases} \\
& d\left(\rho_{\psi+\omega} \rho_{\psi+\omega+1}, \rho_{\lfloor\psi+3 / 2\rfloor}\right)=\left\{\begin{array}{l}
\left\lfloor\frac{\psi+1}{2}\right\rfloor-\omega+1, \quad \text { if } \omega=1,2, \ldots,\left\lfloor\frac{\psi+1}{2}\right\rfloor ; \\
\omega-\left\lfloor\frac{\psi+3}{2}\right\rfloor+1, \quad \text { if } \omega=\left\lfloor\frac{\psi+3}{2}\right\rfloor, \ldots, \psi-1,
\end{array}\right. \\
& d\left(\rho_{\omega} \rho_{\psi+\omega}, \rho_{\psi+1}\right)= \begin{cases}\omega-1, & \text { if } \omega=1,2, \ldots,\left\lfloor\frac{\psi+2}{2}\right\rfloor ; \\
\left\lfloor\frac{\psi+4}{2}\right\rfloor-\omega+\left\lfloor\frac{\psi-1}{2}\right\rfloor, & \text { if } \omega=\left\lfloor\frac{\psi+4}{2}\right\rfloor, \ldots, \psi,\end{cases} \\
& d\left(\rho_{\omega} \rho_{\omega+1}, \rho_{\psi+1}\right)= \begin{cases}\omega, & \text { if } \omega=1,2, \ldots,\left\lfloor\frac{\psi}{2}\right\rfloor ; \\
\left\lfloor\frac{\psi-1}{2}\right\rfloor-\omega+\left\lfloor\frac{\psi+2}{2}\right\rfloor, & \text { if } \omega=\left\lfloor\frac{\psi+2}{2}\right\rfloor, \ldots, \psi,\end{cases} \\
& d\left(\rho_{\psi+\omega} \rho_{\psi+\omega+1}, \rho_{\psi+1}\right)= \begin{cases}\omega-1, & \text { if } \omega=1,2, \ldots,\left\lfloor\frac{\psi+2}{2}\right\rfloor ; \\
\left\lfloor\frac{\psi-1}{2}\right\rfloor-\omega+\left\lfloor\frac{\psi+4}{2}\right\rfloor, & \text { if } \omega=\left\lfloor\frac{\psi+4}{2}\right\rfloor, \ldots, \psi-1,\end{cases} \\
& d\left(\rho_{2 \psi} \rho_{1}, \rho_{\psi+1}\right)=1 \text {. }
\end{aligned}
$$


From the above given representation of all edges with respect to edge resolving set $Q_{e}$, no two edges have the same representation, and it proved that $\operatorname{dim}_{e}\left(\mathrm{ML}_{\psi}\right) \leq 4$.

Now, on contrary, $\operatorname{dim}_{e}\left(\mathrm{ML}_{\psi}\right)=3$ implies that the cardinality of edge metric generator $Q_{e}^{\prime}$ is three, following is some discussion for this contradiction:

Case 1: if the first two vertices with zero-size gap and last two vertices with any arbitrary size of gap are selected in the edge metric generator $Q_{e}^{\prime}=\left\{\rho_{1}, \rho_{2}, \rho_{\omega}\right\}$ with $3 \leq \omega \leq \psi$, then it implies the same representations in the edges $d\left(\rho_{1} \rho_{\psi+1} \mid Q_{e}^{\prime}\right)=d\left(\rho_{1} \rho_{2 \psi} \mid Q_{e}^{\prime}\right)$, and it is concluded that we cannot take these types of vertices in the edge metric generator with cardinality three.

Case 2: if all three vertices with any arbitrary size of gap are selected and $Q_{e}^{\prime}=\left\{\rho_{\omega}, \rho_{j}, \rho_{k}\right\}$ with $1 \leq \omega, j, k \leq \psi$, then it implies the same representations in the edges which are $d\left(\rho_{1} \rho_{\psi+1} \mid Q_{e}^{\prime}\right)=d\left(\rho_{1} \rho_{2 \psi} \mid Q_{e}^{\prime}\right)$, and it is also concluded that we cannot take these types of vertices in the edge metric generator with cardinality three.

Case 3: now, the vertices with index $\psi+2 \leq \omega, j, k \leq 2 \psi$ with any of the gap-size are chosen and $Q_{e}^{\prime}=\left\{\rho_{\omega}, \rho_{j}, \rho_{k}\right\}$, then it implies the same representations in the edges which are $d\left(\rho_{1} \rho_{2} \mid Q_{e}^{\prime}\right)=d\left(\rho_{1} \rho_{\psi+1} \mid Q_{e}^{\prime}\right)$, and again, we conclude that we cannot take these types of vertices in the edge metric generator with cardinality three.

Case 4: now, the vertices $\rho_{\omega}, \rho_{j}, \rho_{k} \in V\left(\mathrm{ML}_{\psi}\right)$ are chosen with any of the size of gap, then there exist an edge from lower horizontal edges and another edge from upper horizontal edges having the same representations to each other with respect to the selected edge metric generator, i.e., $d\left(\rho_{p} \rho_{p+1} \mid Q_{e}^{\prime}\right)=d\left(\rho_{\psi+p} \rho_{\psi+p+1} \mid Q_{e}^{\prime}\right)$ where $1 \leq p \leq \psi-1$, and finally, we concluded that with any gap-size in the edge metric generator with cardinality three is not possible, which implies the contradiction that $\operatorname{dim}_{e}\left(\mathrm{ML}_{\psi}\right) \neq 3$. Moreover, this proves the double inequality which is

$$
\operatorname{dim}_{e}\left(\mathrm{ML}_{\psi}\right)=4
$$

\section{Edge Metric Dimension of Hexagonal Möbius Ladder Network}

Hexagonal Möbius ladder $\mathrm{HML}_{\psi}$ is built in [25], it can be constructed by dividing each horizontal edge of a square grid by inserting a new vertex, it becomes a grid of $\psi \times 1$ with each cycle having order six, and now, twist this grid at $180^{\circ}$ and paste the extreme most left and right paths of vertices as shown in Figure 2. This graph contains $\psi$-horizontal cycles of order six. The metric dimension of the hexagonal Möbius ladder network is three [25]. In this section, we proved that edge metric dimension is also three for the hexagonal Möbius ladder network.

Theorem 2. Let $H M L_{\psi}$ be a hexagonal Möbius ladder network with $\psi \geq 2$. Then,

$$
\operatorname{dim}_{e}\left(\mathrm{HML}_{\psi}\right)=3
$$

Proof. Consider the edge resolving set $Q_{e}=\left\{\rho_{1}, \rho_{2}, \rho_{2 \psi}\right\}$ when $\psi=2,3, Q_{e}=\left\{\rho_{1}, \rho_{\psi+1}, \rho_{2 \psi}\right\}$ when $\psi \geq 4$ (even), and $Q_{e}=\left\{\rho_{1}, \rho_{\psi}, \rho_{2 \psi}\right\}$ when $\psi \geq 5$ (odd). To show that the $\operatorname{dim}_{e}\left(\mathrm{HML}_{\psi}\right)=3$, we will use the method of double inequality. For $\operatorname{dim}_{e}\left(\mathrm{HML}_{\psi}\right) \leq 3$, following are the distances of all edges with respect to edge resolving set.

Distances of all vertices with respect to $\rho_{2}$ are as follows:

$$
\begin{aligned}
d\left(\rho_{\omega} \rho_{\psi+\omega}, \rho_{2}\right)= \begin{cases}1, & \text { if } \omega=1, \text { and } \psi=2 ; \\
\omega-2, & \text { if } \omega=3, \text { and } \psi=2 ; \\
1, & \text { if } \omega=1, \text { and } \psi=3 ; \\
\omega-2, & \text { if } \omega=3,5, \text { and } \psi=3,\end{cases} \\
d\left(\rho_{\omega} \rho_{\omega+1}, \rho_{2}\right)= \begin{cases}0, & \text { if } \omega=1 ; \\
\omega-2, & \text { if } \omega=2,3, \ldots, \psi+2 ; \\
2 \psi+2-\omega, & \text { if } \omega=\psi+3, \psi+4, \ldots, 2 \psi,\end{cases} \\
d\left(\rho_{2 \psi+\omega} \rho_{2 \psi+\omega+1}, \rho_{2}\right)= \begin{cases}2, & \text { if } \omega=1,2 ; \\
\omega-1, & \text { if } \omega=3,4, \ldots, \psi+1 ; \\
2 \psi+2-\omega, & \text { if } \omega=\psi+2, \psi+3, \ldots, 2 \psi-1 .\end{cases} \\
d\left(\rho_{4 \psi} \rho_{1}, \rho_{2}\right)=2, \quad \text { if } \omega=1,3 \text { and } \psi=2 ; \\
d\left(\rho_{4 \psi} \rho_{1}, \rho_{1}\right)=0, \\
d\left(\rho_{\omega} \rho_{\psi+\omega}, \rho_{2 \psi}\right)= \begin{cases}1, & \text { if } \omega=1,5 \text { and } \psi=3 ; \\
1, & \text { if } \omega=1 \\
3, & \text { if } \omega=\psi=3 .\end{cases}
\end{aligned}
$$




$$
\begin{aligned}
& d\left(\rho_{\omega} \rho_{\psi+\omega}, \rho_{1}\right)= \begin{cases}\omega-1, & \text { if } \omega=1,3, \ldots, \psi+1, \text { and } \psi \text { even } \\
2 \psi+1-\omega, & \text { if } \omega=\psi+3, \psi+5, \ldots, 2 \psi-1, \text { and } \psi \text { even } \\
\omega-1, & \text { if } \omega=1,3, \ldots, \psi, \text { and } \psi \text { odd } \\
2 \psi+1-\omega, & \text { if } \omega=\psi+2, \psi+4, \ldots, 2 \psi-1, \text { and } \psi \text { odd }\end{cases} \\
& d\left(\rho_{\omega} \rho_{\omega+1}, \rho_{1}\right)= \begin{cases}\omega-1, & \text { if } \omega=1,2, \ldots, \psi+1 ; \\
2 \psi+1-\omega, & \text { if } \omega=\psi+2, \psi+3, \ldots, 2 \psi .\end{cases} \\
& d\left(\rho_{2 \psi+\omega} \rho_{2 \psi+\omega+1}, \rho_{1}\right)= \begin{cases}\omega, & \text { if } \omega=1,2, \ldots, \psi ; \\
2 \psi-\omega, & \text { if } \omega=\psi+1, \ldots, 2 \psi-1 .\end{cases} \\
& d\left(\rho_{\omega} \rho_{\psi+\omega}, \rho_{\psi}\right)=|\psi-\omega|, \quad \omega=1,3, \ldots, 2 \psi-1, \\
& d\left(\rho_{\omega} \rho_{\omega+1}, \rho_{\psi}\right)= \begin{cases}\psi-\omega-1, & \text { if } \omega=1,2, \ldots, \psi-1 ; \\
\omega-\psi, & \text { if } \omega=\psi, \psi+1, \ldots, 2 \psi .\end{cases} \\
& d\left(\rho_{2 \psi+\omega} \rho_{2 \psi+\omega+1}, \rho_{\psi}\right)= \begin{cases}\psi-\omega, & \text { if } \omega=1,2, \ldots, \psi-1 ; \\
\omega-\psi+1, & \text { if } \omega=\psi, \psi+1, \ldots, 2 \psi-1,\end{cases} \\
& d\left(\rho_{4 \psi} \rho_{1}, \rho_{\psi}\right)=d\left(\rho_{4 \psi} \rho_{1}, \rho_{\psi+1}\right)=\psi, \\
& d\left(\rho_{4 \psi} \rho_{1}, \rho_{2 \psi}\right)=2 \text {, } \\
& d\left(\rho_{\omega} \rho_{\psi+\omega}, \rho_{\psi+1}\right)= \begin{cases}\psi-\omega+1, & \text { if } \omega=1,3, \ldots, \psi+1 ; \\
\omega-\psi-1, & \text { if } \omega=\psi+3, \psi+5, \ldots, 2 \psi-1,\end{cases} \\
& d\left(\rho_{\omega} \rho_{\omega+1}, \rho_{\psi+1}\right)= \begin{cases}\psi-\omega, & \text { if } \omega=1,2, \ldots, \psi ; \\
\omega-\psi-1, & \text { if } \omega=\psi+1, \ldots, 2 \psi .\end{cases} \\
& d\left(\rho_{2 \psi+\omega} \rho_{2 \psi+\omega+1}, \rho_{\psi+1}\right)= \begin{cases}\psi-\omega+1, & \text { if } \omega=1,2, \ldots, \psi ; \\
\omega-\psi, & \text { if } \omega=\psi+1, \psi+2, \ldots, 2 \psi-1,\end{cases} \\
& d\left(\rho_{\omega} \rho_{\psi+\omega}, \rho_{2 \psi}\right)= \begin{cases}1, & \text { if } \omega=1 \\
\omega+1, & \text { if } \omega=3,5, \ldots, \psi-1, \text { and } \psi \geq 4 \text { (even) } \\
2 \psi-\omega, & \text { if } \omega=\psi+1, \psi+3, \ldots, 2 \psi-1, \text { and } \psi \geq 4 \text { (even) } \\
\omega+1, & \text { if } \omega=3,5, \ldots, \psi-2, \text { and } \psi \geq 5 \text { (odd) } \\
2 \psi-\omega, & \text { if } \omega=\psi, \psi+2, \ldots, 2 \psi-1, \text { and } \psi \geq 5 \text { (odd) }\end{cases} \\
& d\left(\rho_{\omega} \rho_{\omega+1}, \rho_{2 \psi}\right)= \begin{cases}\omega+1, & \text { if } \omega=1,2, \ldots, \psi-1 ; \\
2 \psi-1-\omega, & \text { if } \omega=\psi, \psi+1, \ldots, 2 \psi-1 ; \\
0, & \text { if } \omega=2 \psi,\end{cases} \\
& d\left(\rho_{2 \psi+\omega} \rho_{2 \psi+\omega+1}, \rho_{2 \psi}\right)= \begin{cases}\omega+1, & \text { if } \omega=1,2, \ldots, \psi-1 \\
2 \psi-\omega, & \text { if } \omega=\psi, \psi+1, \ldots, 2 \psi-2 \\
2, & \text { if } \omega=2 \psi-1\end{cases}
\end{aligned}
$$

It is clear to see that the representations of all edges with respect to edge resolving set $Q_{e}$ are distinct, and it is proved that $\operatorname{dim}_{e}\left(\mathrm{HML}_{\psi}\right) \leq 3$.

Now, for $\operatorname{dim}_{e}\left(\mathrm{HML}_{\psi}\right) \geq 3$, the contradiction method implies that $\operatorname{dim}_{e}\left(\mathrm{HML}_{\psi}\right)=2$.

If two vertices are chosen with any arbitrary size of gap and $Q_{e}^{\prime}=\left\{\rho_{\omega}, \rho_{j}\right\}$ with $1 \leq \omega, j \leq 4 \psi$, then it implies the same representations in the edges which are either $d\left(\rho_{p} \rho_{\psi+p} \mid Q_{e}^{\prime}\right)=$ $d\left(\rho_{2 \psi+q} \rho_{2 \psi+q+1} \mid Q_{e}^{\prime}\right)$ where $1 \leq p, q \leq 2 \psi-1$ and $p=$ odd or $d\left(\rho_{p} \rho_{p+1} \mid Q_{e}^{\prime}\right)=d\left(\rho_{2 \psi+p} \rho_{2 \psi+p+1} \mid Q_{e}^{\prime}\right)$ where $1 \leq p \leq 2 \psi-1$, and it is also concluded that we cannot take vertices in edge metric generator with cardinality two. So, it is not possible that $\operatorname{dim}_{e}\left(\mathrm{HML}_{\psi}\right)=2$, which implies that $\operatorname{dim}_{e}\left(\mathrm{HML}_{\psi}\right)=3$.

\section{Edge Metric Dimension of Triangular Ladder Network}

The ladder network can be built by the cross product of two path graphs $L_{\psi}=P_{\psi} \times P_{2}$. Triangular ladder $\mathrm{TL}_{\psi}$ is built by inserting new edges of $L_{\psi}$ vertices $\rho_{\omega} \rho_{\omega+1}$ where $\omega$ is even indices. This graph contains $2 \psi-2$-cycles of order three shown in Figure 3. Following is the edge metric generator of this network.

Theorem 3. Let $T L_{\psi}$ be a triangular ladder network with $\psi \geq 3$. Then,

$$
\operatorname{dim}_{e}\left(\mathrm{TL}_{\psi}\right)=4
$$




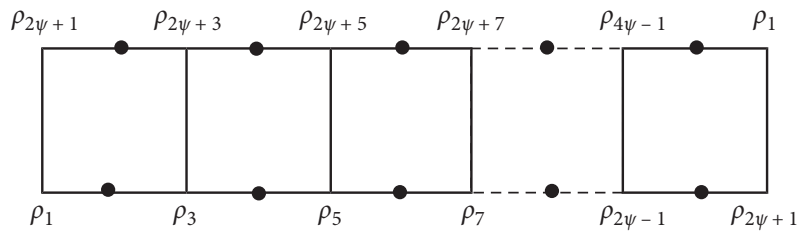

Figure 2: Hexagonal Möbius ladder network $\mathrm{HML}_{\psi}$.

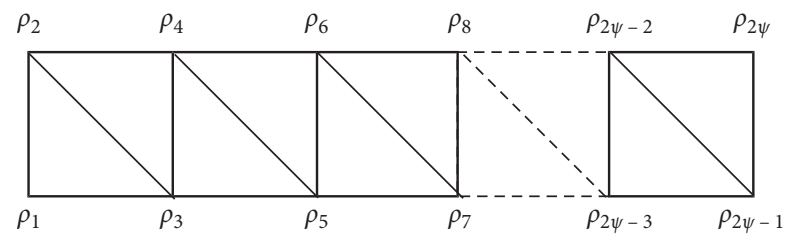

Figure 3: Triangular ladder network $\mathrm{TL}_{\psi}$.

Proof. Let $Q_{e}=\left\{\rho_{1}, \rho_{2}, \rho_{2 \psi-2}, \rho_{2 \psi-1}\right\}$ be the edge resolving set. To show that the $\operatorname{dim}_{e}\left(\mathrm{TL}_{\psi}\right)=4$, we will prove first for $\operatorname{dim}_{e}\left(\mathrm{TL}_{\psi}\right) \leq 4$, following are the representations of all edges with respect to edge resolving set:

$$
\begin{aligned}
d\left(\rho_{\omega} \rho_{\omega+1}, \rho_{1}\right)= \begin{cases}\frac{\omega-1}{2}, & \text { if } \omega=1,3, \ldots, 2 \psi-3 ; \\
\frac{\omega+2}{2}, & \text { if } \omega=2,4, \ldots, 2 \psi-4,\end{cases} \\
d\left(\rho_{\omega} \rho_{\omega+2}, \rho_{1}\right)= \begin{cases}\frac{\omega-1}{2}, & \text { if } \omega=1,3, \ldots, 2 \psi-3 ; \\
\frac{\omega+2}{2}, & \text { if } \omega=2,4, \ldots, 2 \psi-4,\end{cases} \\
d\left(\rho_{\omega} \rho_{\omega+1}, \rho_{2}\right)= \begin{cases}\frac{\omega-1}{2}, & \text { if } \omega=1,3, \ldots, 2 \psi-1 ; \\
d\left(\rho_{\omega} \rho_{\omega+1}, \rho_{2 \psi-2}\right) & = \begin{cases}\frac{\omega-2}{2}, & \text { if } \omega=2,4, \ldots, 2 \psi-2, \\
\frac{2 \psi-\omega}{2}, & \text { if } \omega=2,4, \ldots, 2 \psi-2,\end{cases} \\
\frac{\omega-1}{1,}, & \text { if } \omega=3,5, \ldots, 2 \psi-3 ;\end{cases}
\end{aligned}
$$

$$
\begin{aligned}
& d\left(\rho_{\omega} \rho_{\omega+2}, \rho_{2 \psi-2}\right)= \begin{cases}\frac{2 \psi-\omega-3}{2}, & \text { if } \omega=1,3, \ldots, 2 \psi-5 ; \\
0, & \text { if } \omega=2 \psi-3 ; \\
\frac{\omega-4}{2}, & \text { if } \omega=2 ;\end{cases} \\
& d\left(\rho_{\omega} \rho_{\omega+1}, \rho_{2 \psi-1}\right)= \begin{cases}\frac{2 \psi-\omega-1}{2} & \text { if } \omega=4,6, \ldots, 2 \psi-2, \\
\frac{2 \psi-\omega-2}{2} & \text { if } \omega=2,4, \ldots, 2 \psi-2 ;\end{cases} \\
& d\left(\rho_{\omega} \rho_{\omega+2}, \rho_{2 \psi-1}\right)= \begin{cases}\frac{2 \psi-\omega-1}{2}, & \text { if } \omega=1,3, \ldots, 2 \psi-3 ; \\
\frac{2 \psi-\omega}{2}, & \text { if } \omega=2,4, \ldots, 2 \psi-2 .\end{cases}
\end{aligned}
$$

Above given representations of all edges with respect to edge resolving set $Q_{e}$ are unique, and it is proved that $\operatorname{dim}_{e}\left(\mathrm{TL}_{\psi}\right) \leq 4$. Now, for $\operatorname{dim}_{e}\left(\mathrm{TL}_{\psi}\right) \geq 4$, choose on contrary which implies that $\operatorname{dim}_{e}\left(\mathrm{TL}_{\psi}\right)=3$. Analogously, the cardinality of edge metric generator $Q_{e}^{\prime}$ is three, following is some discussion for this contradiction:

Case 1: if all vertices with any arbitrary size of gap are selected and $Q_{e}^{\prime}=\left\{\rho_{1}, \rho_{\omega}, \rho_{j}\right\}$ with $3 \leq \omega, j$ (odd) $\leq$ $2 \psi-1$, then it implies the same representations in the edges which are $d\left(\rho_{2} \rho_{3} \mid Q_{e}^{\prime}\right)=d\left(\rho_{3} \rho_{4} \mid Q_{e}^{\prime}\right)$, and it is concluded that we cannot take vertices like this in the edge metric generator with cardinality three.

Case 2: if all vertices with any arbitrary size of gap are selected and $Q_{e}^{\prime}=\left\{\rho_{\omega}, \rho_{j}, \rho_{k}\right\}$ with $1 \leq \omega, j, k$ (odd) $\leq$ $2 \psi-1$, then it implies the same representations in the edges which are either $d\left(\rho_{1} \rho_{3} \mid Q_{e}^{\prime}\right)=d\left(\rho_{2} \rho_{3} \mid Q_{e}^{\prime}\right)$ or $d\left(\rho_{p} \rho_{p+1} \mid Q_{e}^{\prime}\right)=d\left(\rho_{q} \rho_{q+2} \mid Q_{e}^{\prime}\right)$ where $2 \leq p, q($ even $) \leq$ $2 \psi$, and it is also concluded that we cannot take vertices in the edge metric generator with cardinality three.

Case 3: if all vertices with any arbitrary size of gap are selected and $Q_{e}^{\prime}=\left\{\rho_{\omega}, \rho_{j}, \rho_{k}\right\}$ with $2 \leq \omega, j, k$ (even) $\leq$ $2 \psi$, then it implies the same representations in the edges which are $d\left(\rho_{1} \rho_{2} \mid Q_{e}^{\prime}\right)=d\left(\rho_{2} \rho_{3} \mid Q_{e}^{\prime}\right)$, and it is also concluded that we cannot take vertices in the edge metric generator with cardinality three.

Case 4: now, the vertices $\rho_{\omega}, \rho_{j}, \rho_{k} \in V\left(\mathrm{TL}_{\psi}\right)$ without choosing the size of gap, then there exist an edge from joining edges and another one edge from upper horizontal edges having the same representation to each other with respect to the decided edge metric generator, i.e., $d\left(\rho_{p} \rho_{p+1} \mid Q_{e}^{\prime}\right)=d\left(\rho_{q} \rho_{q+2} \mid Q_{e}^{\prime}\right)$ where $2 \leq p, q$ (even) $\leq 2 \psi$, and finally, we concluded that we cannot take any type of vertices with any gap-size in the edge metric generator with cardinality three, which implies the contradiction that $\operatorname{dim}_{e}\left(\mathrm{TL}_{\psi}\right) \neq 3$, Moreover, this proves the double inequality which is

$$
\operatorname{dim}_{e}\left(\mathrm{TL}_{\psi}\right)=4
$$




\section{Edge Metric Dimension of Triangular Möbius Ladder Network}

Figure 3 shows a triangular ladder network; now, twist this network at $180^{\circ}$ and paste the extreme most left and right paths of vertices, and it will come up to a new type of graph named as triangular Möbius ladder graph $\mathrm{TML}_{\psi}$. This graph contains $2 \psi-2$-horizontal cycles of order three, which can be seen in Figure 4. Following results are the edge metric dimension of the triangular Möbius ladder network.

Theorem 4. Let $T M L_{\psi}$ be a triangular Möbius ladder network with $\psi \geq 5$. Then,

$$
\operatorname{dim}_{e}\left(\mathrm{TML}_{\psi}\right)=4 .
$$

Proof. Consider the edge resolving set $Q_{e}=\left\{\rho_{4}, \rho_{5}, \rho_{6}, \rho_{7}\right\}$, when $\psi=5, Q_{e}=\left\{\rho_{1}, \rho_{4}, \rho_{\psi+2}, \rho_{\psi+3}\right\}$, when $\psi \geq 6$ (even), and $Q_{e}=\left\{\rho_{3}, \rho_{\psi-1}, \rho_{\psi+2}, \rho_{2 \psi-2}\right\}$, and when $\psi \geq 7$ (odd). To show that the $\operatorname{dim}_{e}\left(\mathrm{TML}_{\psi}\right)=4$, we will use the method of double inequality, for $\operatorname{dim}_{e}\left(\mathrm{TML}_{\psi}\right) \leq 4$, following are the representations of all edges with respect to the edge resolving set.

When $\psi=5$,

$d\left(\rho_{\omega} \rho_{\omega+1}, \rho_{4}\right)= \begin{cases}1, & \text { if } \omega=1,2, \\ \frac{\omega-3}{2}, & \text { if } \omega=3,5,7, \\ \frac{\omega-4}{2}, & \text { if } \omega=4,6,8,\end{cases}$

$d\left(\rho_{2 \psi-2} \rho_{2}, \rho_{4}\right)=d\left(\rho_{2 \psi-3} \rho_{2}, \rho_{4}\right)=d\left(\rho_{2 \psi-1} \rho_{1}, \rho_{4}\right)=2$,

$d\left(\rho_{\omega} \rho_{\omega+2}, \rho_{4}\right)= \begin{cases}2-\omega, & \text { if } \omega=1,2, \\ \frac{\omega-1}{2}, & \text { if } \omega=3,5, \\ \frac{\omega-4}{2}, & \text { if } \omega=4,6,8,\end{cases}$

$d\left(e_{1}, \rho_{5}\right)= \begin{cases}\left|\frac{5-\omega}{2}\right| & \text { if } \omega=1,3,5,7, \\ \frac{4-\omega}{2} & \text { if } \omega=2,4,6\end{cases}$

$d\left(\rho_{2 \psi-2} \rho_{2}, \rho_{5}\right)=d\left(\rho_{2 \psi-1} \rho_{1}, \rho_{5}\right)=2, d\left(\rho_{2 \psi-3} \rho_{2}, \rho_{5}\right)=1$,

$d\left(\rho_{\omega} \rho_{\omega+2}, \rho_{5}\right)= \begin{cases}\omega ; & \text { if } \omega=1,2, \\ 0 ; & \text { if } \omega=3,5, \\ 1 ; & \text { if } \omega=4,6,\end{cases}$

$d\left(\rho_{\omega} \rho_{\omega+1}, \rho_{6}\right)= \begin{cases}\left|\frac{5-\omega}{2}\right| & \text { if } \omega=1,3,5,7, \\ \left|\frac{6-\omega}{2}\right|, & \text { if } \omega=2,4,6,\end{cases}$

$$
\begin{aligned}
& d\left(\rho_{2 \psi-2} \rho_{2}, \rho_{6}\right)=d\left(\rho_{2 \psi-1} \rho_{1}, \rho_{6}\right)=1, \\
& d\left(\rho_{2 \psi-3} \rho_{2}, \rho_{6}\right)=2, \\
& d\left(\rho_{\omega} \rho_{\omega+2}, \rho_{6}\right)= \begin{cases}\frac{2}{\omega} ; \quad \omega=1,2, \\
1 ; \quad \omega=3,5, \\
0 ; \quad \text { if } \omega=4,6,\end{cases} \\
& d\left(\rho_{\omega} \rho_{\omega+1}, \rho_{7}\right)= \begin{cases}\frac{1 ;}{2} ; & \text { if } \omega=3,5,7, \\
\frac{6-\omega}{2} ; & \text { if } \omega=2,4,6,\end{cases} \\
& d\left(\rho_{2 \psi-2} \rho_{2}, \rho_{7}\right)=d\left(\rho_{2 \psi-1} \rho_{1}, \rho_{7}\right)=1, \\
& d\left(\rho_{2 \psi-3} \rho_{2}, \rho_{6}\right)=0, \quad \text { if } \omega=6 .
\end{aligned}
$$

When $\psi \geq 6$ (even),

$$
\begin{aligned}
& d\left(\rho_{\omega} \rho_{\omega+1}, \rho_{1}\right)= \begin{cases}\frac{\omega-2}{2} ; & \text { if } \omega=1,3, \ldots, \psi-1, \\
\frac{2 \psi-\omega-3}{2} ; & \text { if } \omega=\psi+1, \psi+3, \ldots, 2 \psi-3, \\
d\left(\rho_{2 \psi-2} \rho_{2}, \rho_{1}\right)=d\left(\rho_{2 \psi-3} \rho_{2}, \rho_{1}\right)=1, \\
d\left(\rho_{2 \psi-1} \rho_{1}, \rho_{1}\right)=0, & \text { if } \omega=2,4, \ldots, \psi, \\
\frac{2 \psi-\omega}{2} ; & \text { if } \omega=\psi+2, \psi+4, \ldots, 2 \psi-4, \\
\frac{\omega-2}{2} ; & \text { if } \omega=1,3, \ldots, \psi-1, \\
\frac{2 \psi-\omega-1}{2} ; & \text { if } \omega=\psi+1, \psi+3, \ldots, 2 \psi-5, \\
d\left(\rho_{\omega} \rho_{\omega+2}, \rho_{1}\right)=\{ & \text { if } \omega=2,4, \ldots, \psi-2, \\
\frac{\omega}{2} ; & \text { if } \omega=\psi, \psi+2, \ldots, 2 \psi-4, \\
\frac{2 \psi-\omega-2}{2} & \end{cases}
\end{aligned}
$$




$$
\begin{aligned}
& d\left(\rho_{\omega} \rho_{\omega+1}, \rho_{4}\right)= \begin{cases}1 ; & \text { if } \omega=1,2, \\
\frac{\omega-3}{2} ; & \text { if } \omega=3,5, \ldots, \psi+3, \\
\frac{2 \psi-\omega+3}{2} ; & \text { if } \omega=\psi+5, \psi+7, \ldots, 2 \psi-3 \\
\frac{\omega-4}{2} ; & \text { if } \omega=4,6, \ldots, \psi+2, \\
\frac{2 \psi-\omega+2}{2} ; & \text { if } \omega=\psi+4, \psi+6, \ldots, 2 \psi-4 .\end{cases} \\
& d\left(\rho_{2 \psi-2} \rho_{2}, \rho_{4}\right)=d\left(\rho_{2 \psi-3} \rho_{2}, \rho_{4}\right)=d\left(\rho_{2 \psi-1} \rho_{1}, \rho_{4}\right)=2 .
\end{aligned}
$$

When $\psi \geq 7$ (odd),

$$
\begin{aligned}
& d\left(\rho_{\omega} \rho_{\omega+2}, \rho_{4}\right)= \begin{cases}\frac{2-\omega ;}{2} ; & \text { if } \omega=1,2, \\
\frac{2 \psi-\omega+1}{2} ; & \text { if } \omega=\psi+3,5, \ldots, \psi+1, \\
\frac{\omega-4}{2} ; & \text { if } \omega=4,6, \ldots, \psi+2, \\
\frac{2 \psi-\omega+2}{2} ; & \text { if } \omega=\psi+4, \psi+6, \ldots, 2 \psi-4,\end{cases} \\
& d\left(\rho_{\omega} \rho_{\omega+1}, \rho_{\psi+2}\right)= \begin{cases}\frac{\psi-2}{2} ; & \text { if } \omega=1, \\
\left|\frac{\psi-\omega+1}{2}\right| ; & \text { if } \omega=3,5, \ldots, 2 \psi-3, \\
\left|\frac{\psi-\omega-2}{2}\right| ; & \text { if } \omega=2,4, \ldots, 2 \psi-4,\end{cases} \\
& d\left(\rho_{2 \psi-2} \rho_{2}, \rho_{\psi+2}\right)=d\left(\rho_{2 \psi-3} \rho_{2}, \rho_{\psi+2}\right)=d\left(\rho_{2 \psi-1} \rho_{1}, \rho_{\psi+2}\right)=\frac{\psi-4}{2}, \\
& d\left(\rho_{\omega} \rho_{\omega+2}, \rho_{\psi+2}\right)= \begin{cases}\frac{\psi-\omega+1}{2} ; & \text { if } \omega=1,3, \ldots, \psi-1, \\
\frac{\psi-\omega+3}{2} ; & \text { if } \omega=\psi+1, \psi+3, \ldots, 2 \psi-5, \\
\frac{\psi-\omega}{2} ; & \text { if } \omega=2,4, \ldots, \psi, \\
\frac{\omega-\psi-2}{2} ; & \text { if } \omega=\psi+2, \psi+4, \ldots, 2 \psi-4,\end{cases} \\
& d\left(\rho_{\omega} \rho_{\omega+1}, \rho_{\psi+3}\right)= \begin{cases}\frac{\psi^{2}-4}{2} ; & \text { if } \omega=1, \\
\left|\frac{\psi-\omega+3}{2}\right| ; & \text { if } \omega=3,5, \ldots, 2 \psi-3, \\
\frac{\psi-2}{2} ; & \text { if } \omega=2, \\
\left|\frac{\psi-\omega+4}{2}\right| ; & \text { if } \omega=4,6, \ldots, 2 \psi-4,\end{cases}
\end{aligned}
$$

$$
\begin{aligned}
& d\left(\rho_{2 \psi-2} \rho_{2}, \rho_{\psi+3}\right)=d\left(\rho_{2 \psi-1} \rho_{1}, \rho_{\psi+3}\right)=\frac{\psi-4}{2}, \\
& d\left(\rho_{2 \psi-3} \rho_{2}, \rho_{\psi+3}\right)=\frac{\psi-6}{2}, \\
& d\left(\rho_{\omega} \rho_{\omega+2}, \rho_{\psi+3}\right)= \begin{cases}\frac{\psi-2}{2} ; & \text { if } \omega=1,2, \\
\frac{\psi-\omega+2}{2} ; & \text { if } \omega=4,6, \ldots, \psi, \\
\frac{\psi-\omega+1}{2}, & \text { if } \omega=3,5, \ldots, 2 \psi-5, \\
\frac{\omega-\psi-2}{2} ; & \text { if } \omega=\psi+4, \psi+6, \ldots, 2 \psi-4 .\end{cases}
\end{aligned}
$$

It is clear to see the distinct representations of all edges with respect to edge resolving set $Q_{e}$, and it is proved that $\operatorname{dim}_{e}\left(\mathrm{TML}_{\psi}\right) \leq 4$.

Now, for $\operatorname{dim}_{e}\left(\mathrm{TML}_{\psi}\right) \geq 4$, choose the contradiction method, and it implies that $\operatorname{dim}_{e}\left(\mathrm{TML}_{\psi}\right)=3$. Now, the cardinality of edge metric generator $Q_{e}^{\prime}$ is three, following is some discussion for this contradiction:

Case 1: if three vertices with any arbitrary size of gap are selected and $Q_{e}^{\prime}=\left\{\rho_{\omega}, \rho_{j}, \rho_{k}\right\}$ with $1 \leq \omega, j, k$ (odd) $\leq 2 \psi-3$, then it implies the same distances in the edges which are either $d\left(\rho_{1} \rho_{2} \mid Q_{e}^{\prime}\right)=d\left(\rho_{1} \rho_{2 \psi-1} \mid Q_{e}^{\prime}\right)$, and it is also concluded that we cannot take vertices in the edge metric generator with cardinality three.

Case 2: if all vertices with any arbitrary size of gap can choose such as $Q_{e}^{\prime}=\left\{\rho_{\omega}, \rho_{j}, \rho_{k}\right\}$ with $2 \leq \omega, j, k$ (even) $\leq 2 \psi-2$, then it implies the same distances in the edges which are $d\left(\rho_{1} \rho_{2} \mid Q_{e}^{\prime}\right)=d\left(\rho_{2} \rho_{3} \mid Q_{e}^{\prime}\right)$, and it is also concluded that we cannot take vertices in the edge metric generator with cardinality three.

Case 3: now, the vertices $\rho_{\omega}, \rho_{j}, \rho_{k} \in V\left(\mathrm{TML}_{\psi}\right)$ without choosing the size of gap, then there exist an edge from joining edges and another one edge from upper horizontal edges having the same distances to each other with respect to the decided edge metric generator, i.e., $d\left(\rho_{p} \rho_{p+1} \mid Q_{e}^{\prime}\right)=d\left(\rho_{p} \rho_{p+2} \mid Q_{e}^{\prime}\right)$ where $2 \leq p($ even $) \leq 2 \psi$ -4 or $d\left(\rho_{p} \rho_{p+2} \mid Q_{e}^{\prime}\right)=d\left(\rho_{q} \rho_{q+1} \mid Q_{e}^{\prime}\right)$ where $1 \leq p($ odd $)$ $\leq 2 \psi-5$, and finally, we concluded that we cannot take any type of vertices with any gap-size in the edge metric generator with cardinality three, which implies the contradiction that $\operatorname{dim}_{e}\left(\mathrm{TML}_{\psi}\right)$ $\neq 3$, Moreover, this proves the double inequality which is

$$
\operatorname{dim}_{e}\left(\mathrm{TML}_{\psi}\right)=4
$$




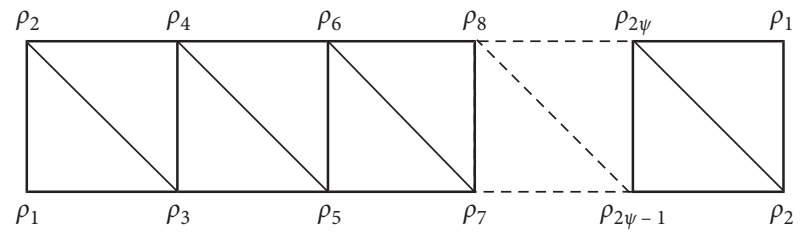

FIgURE 4: Triangular Möbius ladder network $\mathrm{TML}_{\psi}$.

\section{Conclusion}

In the response of the question proposed by Kelenc et al. [3], in this article, we discussed some networks which have $\operatorname{dim}(G)=\operatorname{dim}_{e}(G)$. Hexagonal Möbius ladder has three metric and edge metric dimensions. Triangular ladder, triangular Möbius ladder, and Möbius ladder fall in the category which have $\operatorname{dim}(G)<\operatorname{dim}_{e}(G)$. Moreover, there is no change in the metric dimension and edge metric dimension of triangular ladder graph after making it triangular Möbius ladder and have $\operatorname{dim}_{e}(G)=\operatorname{dim}(G)+1=4$.

\section{Data Availability}

The data used to support the findings of this study are included within the manuscript.

\section{Conflicts of Interest}

The authors declare that they have no conflicts of interest to report regarding the present study.

\section{Acknowledgments}

This study was supported by the NSFQH 11(no. 2018-ZJ925Q).

\section{References}

[1] P. J. Slater, "Leaves of trees, proceeding of the 6th southeastern conference on combinatorics, graph theory, and computing," Congressus Numerantium, vol. 14, pp. 549-559, 1975.

[2] F. Harary and R. A. Melter, "On the metric dimension of a graph,” Ars Combinatoria, vol. 2, pp. 191-195, 1976.

[3] A. Kelenc, N. Tratnik, and I. G. Yero, "Uniquely identifying the edges of a graph: the edge metric dimension," Discrete Applied Mathematics, vol. 251, pp. 204-220, 2018.

[4] G. Chartrand, L. Eroh, M. A. Johnson, and O. R. Oellermann, "Resolvability in graphs and the metric dimension of a graph," Discrete Applied Mathematics, vol. 105, no. 1-3, pp. 99-113, 2000.

[5] S. Khuller, B. Raghavachari, and A. Rosenfeld, "Landmarks in graphs," Discrete Applied Mathematics, vol. 70, no. 3, pp. 217-229, 1996.

[6] V. Chvátal, "Mastermind," Combinatorica, vol. 3, no. 3-4, pp. 325-329, 1983.

[7] P. Erdös and P. R. Alfréd, "On two problems of information theory," Matematikai Kutato Intezetenek Kozlemenyei. Magyar Tudomanyos Akademia.vol. 8, pp. 229-243, 1963.

[8] B. Lindström, "On a combinatory detection problem," Matematikai Kutato Intezetenek Kozlemenyei. Magyar Tudomanyos Akademia.vol. 9, pp. 195-207, 1964.
[9] M. Ali, G. Ali, M. Imran, A. Q. Baig, and M. K. Shafiq, "On the metric dimension of Möbius ladders," Ars Combinatoria, vol. 105, pp. 403-410, 2012.

[10] M. Ali, G. Ali, U. Ali, and M. T. Rahim, "On cycle related graphs with constant metric dimension," Open Journal of Discrete Mathematics, vol. 2, no. 1, pp. 21-23, 2012.

[11] D. Kuziak, J. A. Rodríguez-Velázquez, and I. G. Yero, "On the strong metric dimension of product graphs," Electronic Notes in Discrete Mathematics, vol. 46, pp. 169-176, 2014.

[12] J.-B. Liu, M. F. Nadeem, H. M. A. Siddiqui, and W. Nazir, "Computing metric dimension of certain families of Toeplitz graphs," IEEE Access, vol. 7, pp. 126734-126741, 2019.

[13] J.-B. Liu, A. Zafari, and H. Zarei, "Metric dimension, minimal doubly resolving sets, and the strong metric dimension for jellyfish graph and cocktail party graph," Complexity, vol. 2020, Article ID 9407456, 7 pages, 2020.

[14] A. Ahmad, M. Bača, and S. Sultan, "Computing the metric dimension of kayak paddles graph and cycles with chord," Proyecciones (Antofagasta), vol. 39, no. 2, pp. 287-300, 2020.

[15] A. N. A. Koam and A. Ahmad, "Barycentric subdivision of Cayley graphs with constant edge metric dimension," IEEE Access, vol. 8, pp. 80624-80628, 2020.

[16] Y. Zhang and S. Gao, "On the edge metric dimension of convex polytopes and its related graphs," Journal of Combinatorial Optimization, Springer, vol. 39, no. 2, pp. 334-350, 2020.

[17] M. Ahsan, Z. Zahid, S. Zafar, A. Rafiq, M. Sarwar Sindhu, and M. Umar, "Computing the edge metric dimension of convex polytopes related graphs," Journal of Mathematics and Computer Science, vol. 22, no. 2, pp. 174-188, 2020.

[18] B. Yang, M. Rafiullah, H. M. A. Siddiqui, and S. Ahmad, "On resolvability parameters of some wheel-related graphs," Journal of Chemistry, vol. 2019, Article ID 9259032, 9 pages, 2019.

[19] Z. Raza and M. S. Bataineh, "The comparative analysis of metric and edge metric dimension of some subdivisions of the wheel graph," Asian-European Journal of Mathematics, vol. 14, no. 4, p. 2150062, 2020.

[20] F. Okamoto, B. Phinezy, and P. Zhang, "The local metric dimension of a graph," Mathematica Bohemica, vol. 135, no. 3, pp. 239-255, 2010.

[21] I. G. Yero, "Vertices, edges, distances and metric dimension in graphs," Electronic Notes in Discrete Mathematics, vol. 55, pp. 191-194, 2016.

[22] H. Raza, J.-B. Liu, and S. Qu, "On mixed metric dimension of rotationally symmetric graphs," IEEE Access, vol. 8, pp. 11560-11569, 2020.

[23] H. Raza and Y. Ji, "Computing the mixed metric dimension of a generalized petersen graph $P(n, 2)$," Frontiers in Physics, vol. 8, p. 211, 2020.

[24] H. Raza, Y. Ji, and S. Qu, "On mixed metric dimension of some path related graphs," IEEE Access, vol. 8, pp. 188146$188153,2020$.

[25] M. F. Nadeem, M. Azeem, and A. Khalil, "The locating number of hexagonal Möbius ladder network," Journal of Applied Mathematics and Computing, 2020. 